\title{
A Retrial Queueing model with FDL at OBS core node
}

\author{
Chuong Dang Thanh ${ }^{\mathrm{a}}$, Duc Pham Trung ${ }^{\mathrm{a}}$, Thang Doan Van ${ }^{\mathrm{b}}$ \\ aFaculty of Information Technology, College of Sciences, Hue University, \\ Hue, Viet Nam. \\ E-mail: dtchuong@gmail.com \\ bIndustrial University of Ho Chi Minh, Ho ChiMinh, VietNam. \\ E-mail: vanthangdn@gmail.com
}

Received: July 27, $2018 \quad$ Accepted: October 27, $2018 \quad$ Published: October 31, 2018

DOI: 10.5296/npa.v10i3.13431

URL: https:// doi.org/10.5296/npa.v10i3.13431

\begin{abstract}
Optical Burst Switching networks are considered as an important candidate for the future transport networks. Many analysis models of OBS node with FDLs have been proposed recently. In this paper, we propose a novel retrial queueing model at OBS core node architecture SPL - feed-forward. Blocking probability will be calculated based on Markov multi-dimensional models. Numerical solution values from the proposed analysis method are compared with simulation, as well as between these models.
\end{abstract}

Keywords: OBS, Blocking probability, Complete Wavelength Conversion (CWC), Share-Per- Link (SPL), Fiber Delay Lines (FDL), feed-forward, Retrial Queueing. 


\section{Introduction}

Optical Burst Switching (OBS) in the WDM (Wavelength Division Multiplexing) network has been seen as a promising technology for the next generation of the Internet, because it has many attractive advantages such as fast speed and high bandwidth utilization compared to other optical circuit switching models. At the ingress edge node of the OBS network, packets that arrive from the higher layer (such as IP flows) with the same destination (and the same QoS service layer) are aggregated in optical burst, scheduled and sent into the OBS network following a control packet BCP (Burst Control Packet) for a period "offset time". The offset time allows for the control packet to be processed and the switch to be set up before the burst arrives at the intermediate node; thus, no electronic or optical buffering is necessary at the intermediate nodes while the control packet is being processed. By that way, OBS network has eliminated the requirement to use optical buffers, one of the limitations that optical technology is currently unable to get over. At the core nodes the OBS network, a burst is forwarded towards the target node as configured. When the burst data arrives at the egress node after offset time, the burst will be processed in the optical domain entirely, and IP streams will be restored (burst disassembly) from this optical burst and send the packets to the upper network layers [1][2][3].

In the network of switched optical based on the packet (OPS and OBS), a contention will occur when two arriving bursts in an OBS core node simultaneously require the same wavelength on the same output port. If the wavelength of an incoming burst is occupied at the output port when the burst arrives, the burst can be switched to another wavelength (using wavelength conversion). In case all wavelength channels at an output port are occupied, burst arrival can use fiber delay lines (FDLs) to resolve contention. An FDL causes a fixed amount of delay to the signal transmitted over it, and thus an appropriate combination of FDL might be used as a buffer with limited capabilities. However, different from electronic buffers, in the optical network the bursts cannot wait for an indefinite amount of time in an FDL buffer (exceeds allowable of the delay for the optical network). Thus, the bursts in the FDL's may get lost after a certain period of waiting for service [4][5]. Retrial queuing systems with FDL been studied in the literature [4] and [5]. In [5], authors combine the MMPP-based overflow traffic models of the classical circuit switching literature and fixed-point iterations to devise an algorithmic procedure to accurately estimate blocking probabilities as a function of various buffer parameters in the system when burst arrivals are Poisson and burst lengths are exponentially distributed. Model analysis in [4] the use of the $\mathrm{MM} \sum_{k=1}^{K} C P P_{k} / \mathrm{GE} / \mathrm{c} / \mathrm{L} G-$ queue to model nodes, with $c$ servers, Generalized Exponential (GE) service times and with $K$ independent customer arrival streams, each of which is a Compound Poisson Process (CPP), i.e. a Poisson point process with batch arrivals of geometrically distributed batch size.

In this paper, we propose a retrial queuing model $M / M / \omega / \omega+L$ retrial queue comprises a $\omega$-server queuing system for $L$ FDL buffer optical take to consider the factors retrial of the bursts in asynchronous optical switching nodes. This is also different from the analysis model in the [4] and [5]. Accordingly, the characteristics of the FDL analyzed above 
can be considered as features have or not have the patience of customers in the retrial queue. We study new generic queuing model for FDL buffers based on model analyzed in [6] with consideration of the retrial factor, it means that the burst cannot be fed to the FDL when encountered congestion with a probability value $\theta$ or $\theta_{1}$. A burst is called to be a retrial burst once it traverses one of the $L$ FDL. A retrial burst will use one of the free wavelength channels if available at the epoch of exit from the FDL. Details of the analysis model will be presented in the next section of the article.

The rest of this paper is organized as follows: Section part II, a model of performance analysis of OBS core nodes with different traffics will be considered. The results and analysis, combined with simulation will be mentioned in Section III.

\section{A Model of Performance Analysis}

\subsection{The Assumptions}

The same as in [6], Markov model will also be used to evaluate the performance of OBS core nodes. The model is based on the following assumptions:

- The traffic distribution to the output ports is the same, so we just need to look at an output port.

- An OBS core nodes SPL architecture has $K$ input ports and $K$ output ports (Figure 1), each corresponds to an optical fiber carrying $\omega$ wavelengths $\Lambda=$ $\left\{\lambda_{0}, \lambda_{1}, \cdots, \lambda_{\omega-1}\right\}$ (Assuming the wavelength conversion capacity is complete, there will be $\omega$ the $\mathrm{CWC}$ converter on each output port).

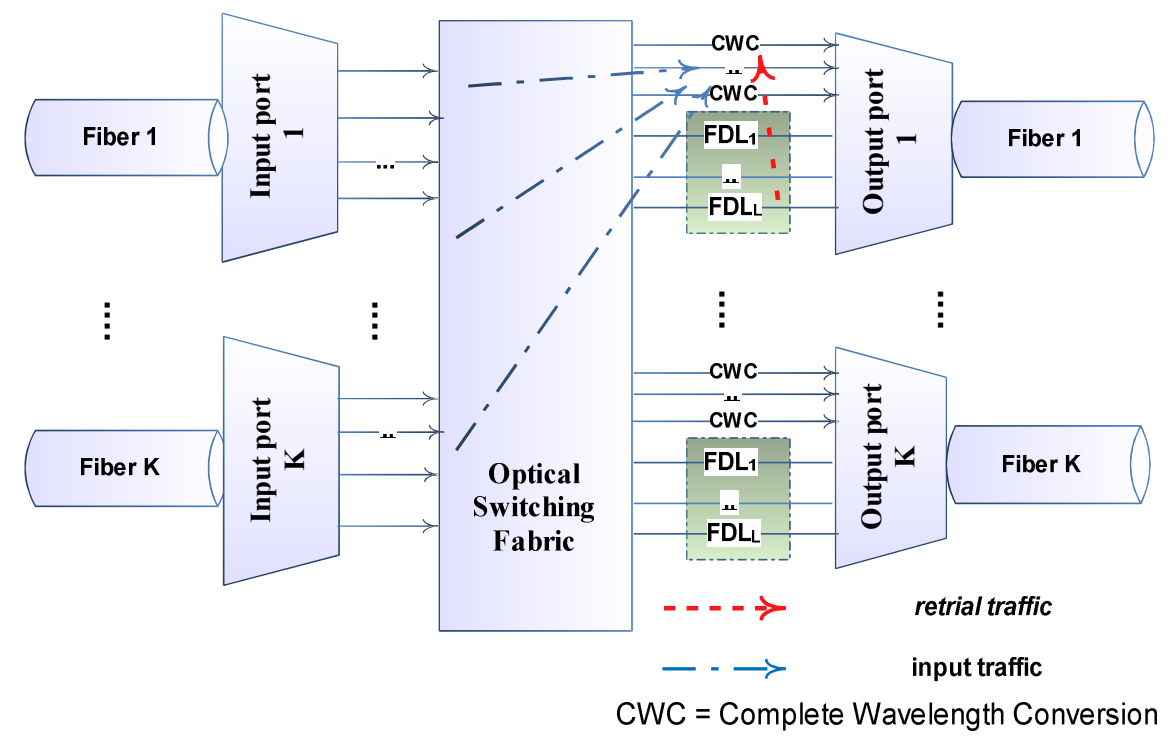

Figure 1. An SPL-OBS core node with FDL

- FDL buffers can also be classified based on their length; FDL architecture with 
fixed length F-FDL use $L$ FLDs the same length and thus all FDLs generate same the delay $D[4][7]$.

- Each incoming burst will be sent to output port, with or without wavelength conversion, depending on the availability of the expected wavelength at the arrival time of the burst.

- In the model, we analysis a burst to the output port at the time all $\omega$ wavelengths are used is thought to be congested. A congested burst will always be able to use a buffer FDL (if available) to retry entry into the output port.

- A burst is said to be a retrial burst once it traverses one of the $L$ FDLs. A retrial burst will re-use one of the free wavelength channels if available at the epoch of exit from the FDL. In this model, we study the queuing model for FDL optical buffers take to consider the factors retrial of the bursts (Figure 2). This is also different from analysis model in [6].

\subsection{Analytical Model with the Retrial Queuing System}

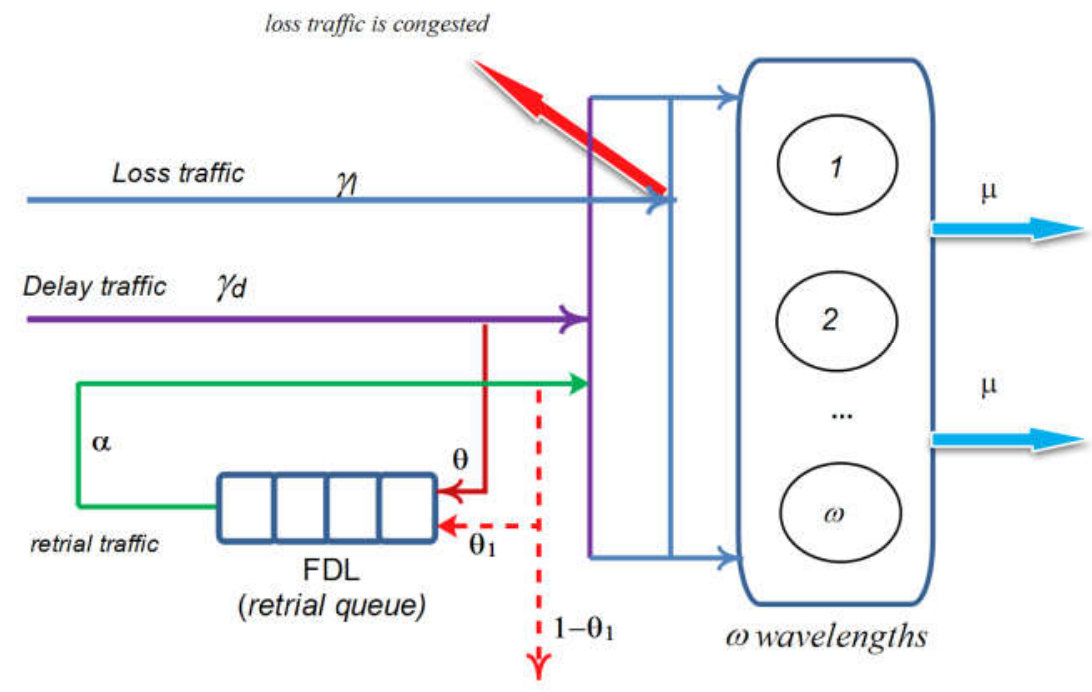

Figure 2. The analysis model retrial queue with FDL feed-forward

Without loss of generality, if we consider at output (Figure. 1), for example, output $k(1 \leq k \leq K)$, can be analyzed as follows: when the traffic arrives to the output port $\mathrm{k}$, if there is a conflict, there are two possible cases: (i) The burst will be dropped due to the lack of wavelength resources (as well as sets CWC), called the lost burst, or (ii) burst will be delayed in the FDL with a certain period of time and continue to share wavelength resources at the epoch of exit from the FDLs (the burst will be dropped when all FDLs are busy or with probability $(1-\theta)$ or $\left.\left(1-\theta_{1}\right)\right)$, called burst delay. In this paper, as mentioned above, the difference between our proposed models versus the model in [6] is that: delayed traffic arrive 
to FDL (orbit queue) with probability $\theta(\theta \leq 1)$ and retrial traffic (bursts come out from the FDL) when continuing the congestion at the output port can also re-circulate through one of the FDLs (2nd) with probability $\theta_{1}\left(\theta_{1}<1\right)$.

\subsubsection{The State Transition Diagram}

This model corresponds to an analysis case with all bursts arrive according to a Poisson flow and service times are exponential. So, the model has the form $M / M / \omega / \omega+L$ retrial queue comprises a $\omega$-server queuing system is described in Figure 2 [7][8][9]. Whereby, the inter-arrival times of loss and delay bursts are exponentially distributed with rates is $\gamma_{l}$ and $\gamma_{d}$, respectively; therefore, the traffic load is $\rho=\rho_{1}+\rho_{2}$, which $\rho_{1}=\gamma_{l} / \mu$ is the traffic load average on the loss bursts and $\rho_{2}=\gamma_{d} / \mu \gamma_{l} / \mu$ is the traffic load average on the delay bursts.

The state transition diagram is illustrated in Figure 3. Each state of this model corresponds to the pair $\{X(t), Y(t): t \geq 0\}$, where $X(t)$ is the number of used wavelengths at time $\mathrm{t}$ and $Y(t)$ is the number of delay burst congested in the FDL (retrial queue). The state space of the CTMC process (symbol S) is described below: $S=\{i, j\}$ with each pair $(i, j)$ identified: $i=0,1,2, \ldots, \omega ; j=0,1,2, \ldots, L$. We denote the steady state probabilities by $\pi_{i, j}=\lim _{t \rightarrow \infty} P(I(t)=i, J(t)=j)$ that the system achieves in state $(i, j)$.

- According to the analysis model in Figure 2 the delay bursts when is congested will be put into the queue FDL with probability $\theta(\theta \leq 1)$, called the retrial burst, and will re-use a wavelength channel if (wavelength) is available at the time the retrial burst exits from the FDL. Otherwise, it will attempt to re-circulate through one of the FDLs (again) with probability $\theta_{1}\left(\theta_{1} \leq 1\right)$ or will be discarded, i.e., lost, with probability $1-\theta_{1}\left(\left(1-\theta_{1}\right)\right.$ is the probability that the burst will leave the system forever).

- The interval-time between the retrial bursts also follows the exponential distribution and the retrial rate is $\alpha$. 


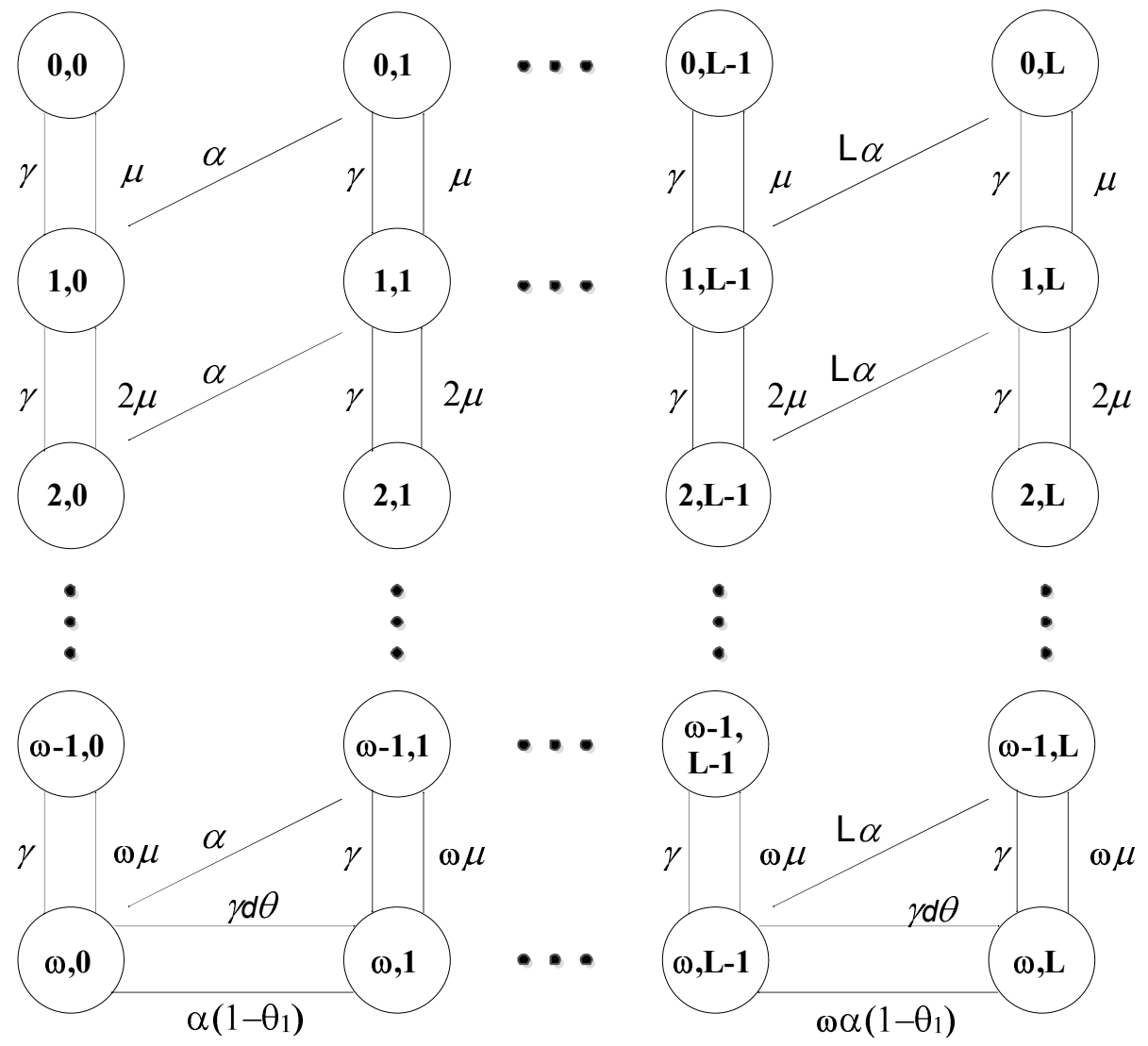

Figure 3. The diagram of all state transitions

Follow the retrial queuing model $M / M / \omega / \omega[10]$, we have matrices with the transition states in Figure 3 as follows:

(a) $\boldsymbol{A}_{\boldsymbol{j}}(\boldsymbol{i}, \boldsymbol{k})$ : denotes the transition rate from state $(i, j)$ to state $(k, j)$ (with $0 \leq i, k \leq$ $\omega ; 0 \leq j \leq L$ ) which is caused by either the departure of a burst after service or the arrival of a burst. Service time is exponentially distributed with parameter $\mu$. Matrix $A_{j}$ has size $(\omega+1) \times(\omega+1)$ with elements $A_{j}(i, k)$. So, $j$ is the independence level of $A_{j}$ so we can be written as $A_{j}=A$. Elements not equal to 0 of $A_{j}$ is

$$
\begin{gathered}
A_{j}(i, i-1)=i \mu, i=\overline{1, \omega+1} \text { and } A_{j}(i, i+1)=\gamma, i=\overline{0, \omega} . \\
A_{j}=A=\left(\begin{array}{ccccccc}
0 & \gamma & 0 & \cdots & 0 & 0 & 0 \\
\mu & 0 & \gamma & \cdots & 0 & 0 & 0 \\
\vdots & \vdots & \vdots & \vdots & \vdots & \vdots & \vdots \\
0 & 0 & 0 & \cdots & (\omega-1) \mu & 0 & \gamma \\
0 & 0 & 0 & \cdots & 0 & \omega \mu & 0
\end{array}\right),(0 \leq j \leq L)
\end{gathered}
$$


(b) $\boldsymbol{B}_{\boldsymbol{j}}(\boldsymbol{i}, \boldsymbol{k})$ : represents one step upward transition from state $(i, j)$ to state $(k, j+1)$ (with $0 \leq i, k \leq \omega ; 0 \leq j \leq L$ ), which is due to a burst joining the orbit (burst come but all wavelength channels are busy (when $i=\omega$ ). In a similar way, matrix $B_{j}$ has size $(\omega+1) \times(\omega+1)$ with elements $B_{j}(i, k)$. Only the non-zero element of $B_{j}$ is $B_{j}(\omega, \omega)=\gamma_{d} \theta$

$$
B_{j}=B=\left(\begin{array}{ccccc}
0 & 0 & \cdots & 0 & 0 \\
0 & 0 & \cdots & 0 & 0 \\
\vdots & \vdots & \vdots & \vdots & \vdots \\
0 & 0 & \cdots & 0 & 0 \\
0 & 0 & \cdots & 0 & \gamma_{d} \theta
\end{array}\right),(0 \leq j \leq L)
$$

(c) $\boldsymbol{C}_{\boldsymbol{j}}(\boldsymbol{i}, \boldsymbol{k})$ : is the transition rate from the state $(i, j)$ to state $(k, j-1)(0 \leq i, k \leq$ $\omega ; 1 \leq j \leq L)$, which is due to a burst which leaves the FDL (orbit). Matrix $C_{j}$ has size $(\omega+1) \times(\omega+1)$ with elements $C_{j}(i, k)$. Elements not equal to 0 of $C_{j}$ is $C_{j}(i, i+1)=j \alpha($ with $i \geq 0)$ and $C_{j}(\omega, \omega)=j \alpha\left(1-\theta_{1}\right)$.

$$
C_{j}=\left(\begin{array}{cccccc}
0 & j \alpha & 0 & \cdots & 0 & 0 \\
0 & 0 & j \alpha & \cdots & 0 & 0 \\
\vdots & \vdots & \vdots & \vdots & \vdots & \vdots \\
0 & 0 & 0 & \cdots & 0 & j \alpha \\
0 & 0 & 0 & \cdots & 0 & j \alpha\left(1-\theta_{1}\right)
\end{array}\right),(1 \leq j \leq L)
$$

As a consequence, the generator matrix $Q$ of the CTMC S is written as:

$$
Q=\left(\begin{array}{ccccc}
Q_{1}^{(0)} & Q_{0}^{(0)} & & & \\
Q_{2}^{(1)} & Q_{1}^{(1)} & Q_{0}^{(1)} & & \\
& Q_{2}^{(2)} & Q_{1}^{(2)} & \ddots & \\
& & \ddots & \ddots & Q_{0}^{(L-1)} \\
& & & Q_{2}^{(L)} & Q_{1}^{(L)}
\end{array}\right)
$$

where 


$$
\left\{\begin{array}{c}
Q_{0}^{(j)}=B(0 \leq j \leq L-1) \\
Q_{2}^{(j)}=C_{j}(1 \leq j \leq L) \\
Q_{1}^{(0)}=A-D^{A}-B \\
Q_{1}^{(j)}=A-D^{A}-B-C_{j}(1 \leq j \leq L-1) \\
Q_{1}^{(L)}=A-D^{A}-C_{j},(j=L)
\end{array}\right.
$$

Call $v_{j}=\left(\pi_{0, j}, \pi_{1, j}, \ldots, \pi_{\omega-1, j}, \pi_{\omega, j}\right)(0 \leq j \leq L)$ and $v=\left(v_{0}, v_{1}, \ldots, v_{L-1}, v_{L}\right)$.

\subsubsection{Balance Equation}

From the state transition diagram and follow (5), the balance equations can be expressed as follows:

$$
\left\{\begin{array}{c}
v_{0} Q_{1}^{(0)}+v_{1} Q_{2}^{(1)}=(0,0, \ldots, 0), \\
v_{j} Q_{0}^{(j)}+v_{j+1} Q_{1}^{(j+1)}+v_{j+2} Q_{2}^{(j+2)}=(0,0, \ldots, 0),(j=\overline{0, L-2}) \\
v_{L-1} Q_{0}^{(L-1)}+v_{L} Q_{1}^{(L)}=(0,0, \ldots, 0) .
\end{array}\right.
$$

In addition, the normalization equation is

$$
\sum_{j=0}^{L} v_{j} e=1
$$

with $e$ is a column vector of size $1 \times(\omega+1)$.

\subsubsection{Calculate the Congestion Probability}

This corresponds to the state diagram in Figure 3, the blocking probability of each loss bursts and delay bursts can be calculated as follows [6]:

- The blocking probability of loss bursts: the loss bursts is congested when all wavelengths are busy at the time they come the system (it corresponds to the states from $(\omega, 0)$ to $(\omega, L)$ in Figure 3).

$$
P B_{\text {loss }}=\sum_{j=0}^{L} \pi_{\omega, j}
$$

- The blocking probability of delay bursts: The delay bursts is congested when all 
wavelength $\omega$ are busy and all locations in the FDL are occupied at the time they come the system.

$$
P B_{\text {delay }}=\frac{\alpha\left(1-\theta_{1}\right)}{\gamma_{d} \theta} \cdot \sum_{j=1}^{L} j \cdot \pi_{\omega, j}
$$

To determine the values $P B_{\text {loss }}$ in equation (8) and $P B_{\text {delay }}$ in equation (9), we must calculate the steady-state probability $\pi_{i, j}$ by solving equation (6) (by the matrix-geometric method, which involves the computation of the rate matrix $Q$ [10][11]).

\subsubsection{Example}

i. Illustration with $\omega=3 ; L=2$

State transition diagram with simple case $\omega=3 ; L=2$ is shown in Figure 4.

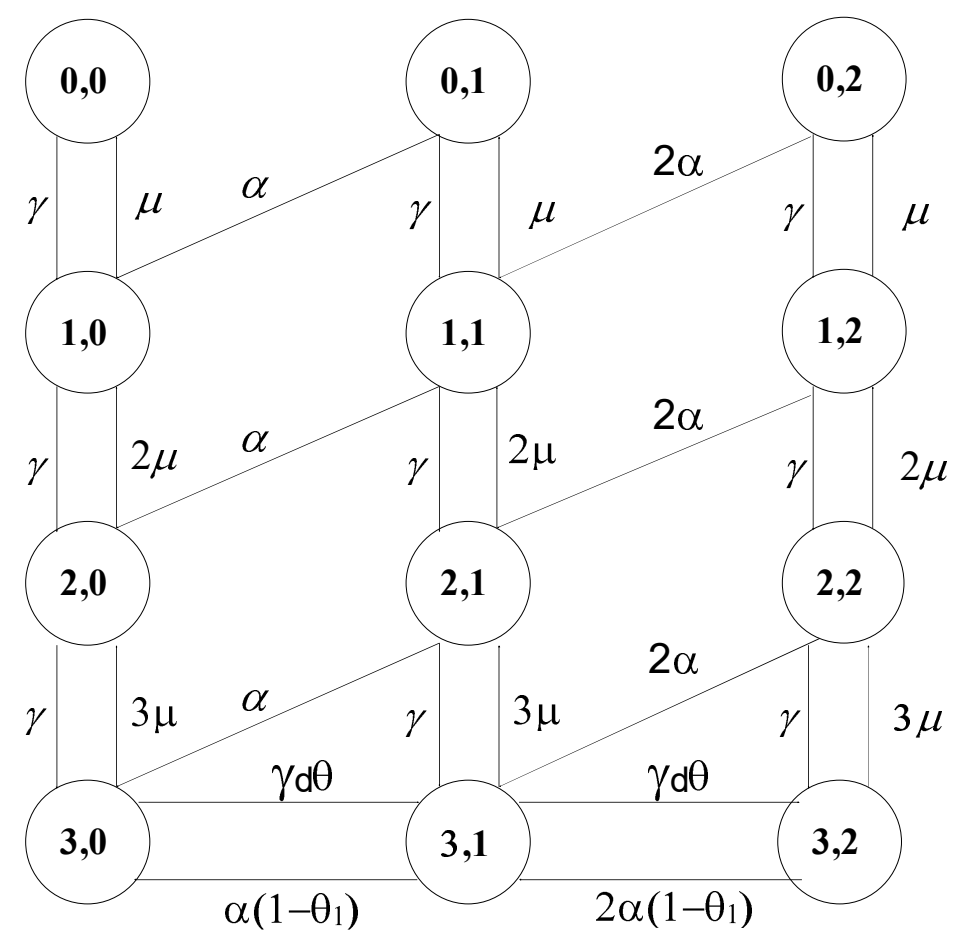

Figure 4. State transition diagram of the analytical model with $\omega=3 ; L=2$

The generator matrix $Q$ is constructed from the matrix $A_{j}(i, k), B_{j}(i, k)$ and $C_{j}(i, k)$ as in Table 1: 


$$
\begin{aligned}
& A=\left(\begin{array}{cccc}
0 & \gamma & 0 & 0 \\
\mu & 0 & \gamma & 0 \\
0 & 2 \mu & 0 & \gamma \\
0 & 0 & 3 \mu & 0
\end{array}\right), D^{A}=\left(\begin{array}{cccc}
\gamma & 0 & 0 & 0 \\
0 & \mu+\gamma & 0 & 0 \\
0 & 0 & 2 \mu+\gamma & 0 \\
0 & 0 & 0 & 3 \mu
\end{array}\right) \\
& B=D^{B}=\left(\begin{array}{cccc}
0 & 0 & 0 & 0 \\
0 & 0 & 0 & 0 \\
0 & 0 & 0 & 0 \\
0 & 0 & 0 & \gamma_{d} \theta
\end{array}\right), Q_{2}^{(2)}=\left(\begin{array}{cccc}
0 & 2 \alpha & 0 & 0 \\
0 & 0 & 2 \alpha & 0 \\
0 & 0 & 0 & 2 \alpha \\
0 & 0 & 0 & 2 \alpha\left(1-\theta_{1}\right)
\end{array}\right) \\
& C_{j}=\left(\begin{array}{cccc}
0 & j \alpha & 0 & 0 \\
0 & 0 & j \alpha & 0 \\
0 & 0 & 0 & j \alpha \\
0 & 0 & 0 & j \alpha\left(1-\theta_{1}\right)
\end{array}\right) \\
& D^{C_{j}}=\left(\begin{array}{cccc}
0 & 0 & 0 & 0 \\
0 & 0 & 0 & 0 \\
0 & 0 & 0 & 0 \\
0 & 0 & 0 & j \alpha\left(1-\theta_{1}\right)
\end{array}\right), Q_{1}^{(0)}=\left(\begin{array}{cccc}
-\gamma & \gamma & 0 & 0 \\
\mu & -\mu-\gamma & \gamma & 0 \\
0 & 2 \mu & -2 \mu-\gamma & \gamma \\
0 & 0 & 3 \mu & -3 \mu-\gamma_{d} \theta
\end{array}\right)
\end{aligned}
$$

\begin{tabular}{|c|c|c|c|c|c|c|c|c|c|c|c|c|}
\hline$(i, j)$ & 0,0 & 1,0 & 2,0 & 3,0 & 0,1 & 1,1 & 2,1 & 3,1 & 0,2 & 1,2 & 2,2 & 3,2 \\
\hline 0,0 & $-\gamma$ & $\gamma$ & & & & & & & & & & \\
\hline 1,0 & $\mu$ & $-\mu-\gamma$ & $\gamma$ & & & & & & & & & \\
\hline 2,0 & & $2 \mu$ & $-2 \mu-\gamma$ & $\gamma$ & & & & & & & & \\
\hline 3,0 & & & $3 \mu$ & $-3 \mu$ & & & & $\gamma_{d} \theta$ & & & & \\
\hline 0,1 & & $\alpha$ & & & $-\gamma-\alpha$ & $\gamma$ & & & & & & \\
\hline 1,1 & & & $\alpha$ & & $\boldsymbol{\mu}$ & $-\mu-\gamma$ & $\gamma$ & & & & & \\
\hline 2,1 & & & & $\alpha$ & & $2 \mu$ & $-2 \mu-\gamma$ & $\gamma$ & & & & \\
\hline 3,1 & & & & $\alpha\left(1-\theta_{1}\right)$ & & & $3 \mu$ & $\begin{array}{l}-3 \mu-\gamma_{d} \theta \\
-\alpha\left(1-\theta_{1}\right)\end{array}$ & & & & $\gamma_{d} \theta$ \\
\hline 0,2 & & & & & & $2 \alpha$ & & & $-\gamma-2 \alpha$ & $\gamma$ & & \\
\hline
\end{tabular}

Table 1. Generator matrix $Q$ with $\omega=3 ; L=2$ 


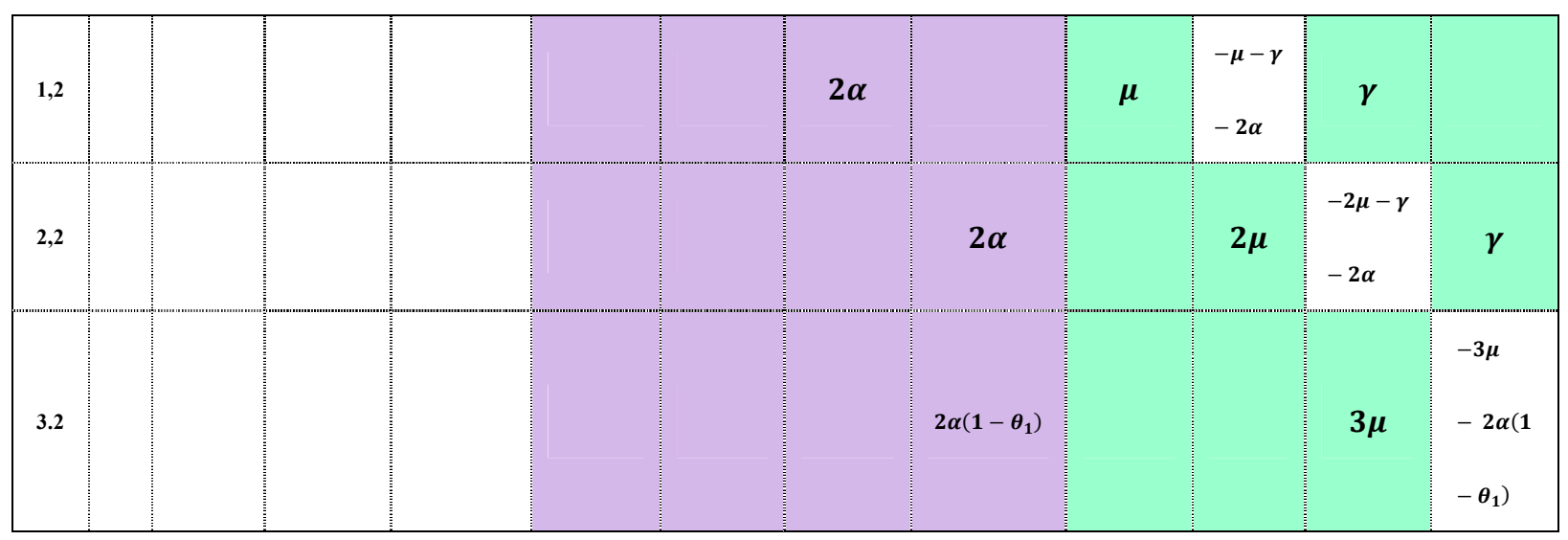

and

$Q_{1}^{(1)}=\left(\begin{array}{cccc}-\gamma-\alpha & \gamma & 0 & 0 \\ \mu & -\mu-\gamma-\alpha & \gamma & 0 \\ 0 & 2 \mu & -2 \mu-\gamma-\alpha & \gamma \\ 0 & 0 & 3 \mu & -3 \mu-\gamma_{d} \theta-\alpha\left(1-\theta_{1}\right)\end{array}\right)$,

$Q_{1}^{(2)}=\left(\begin{array}{cccc}-\gamma-2 \alpha & \gamma & 0 & 0 \\ \mu & -\mu-\gamma-2 \alpha & \gamma & 0 \\ 0 & 2 \mu & -2 \mu-\gamma-2 \alpha & \gamma \\ 0 & 0 & 3 \mu & -3 \mu-2 \alpha\left(1-\theta_{1}\right)\end{array}\right)$,

$Q_{0}^{(0)}=Q_{0}^{(1)}=\left(\begin{array}{cccc}0 & 0 & 0 & 0 \\ 0 & 0 & 0 & 0 \\ 0 & 0 & 0 & 0 \\ 0 & 0 & 0 & \gamma_{d} \theta\end{array}\right), Q_{2}^{(1)}=\left(\begin{array}{cccc}0 & \alpha & 0 & 0 \\ 0 & 0 & \alpha & 0 \\ 0 & 0 & 0 & \alpha \\ 0 & 0 & 0 & \alpha\left(1-\theta_{1}\right)\end{array}\right)$,

$Q_{2}^{(2)}=\left(\begin{array}{cccc}0 & 2 \alpha & 0 & 0 \\ 0 & 0 & 2 \alpha & 0 \\ 0 & 0 & 0 & 2 \alpha \\ 0 & 0 & 0 & 2 \alpha\left(1-\theta_{1}\right)\end{array}\right)$

$Q$

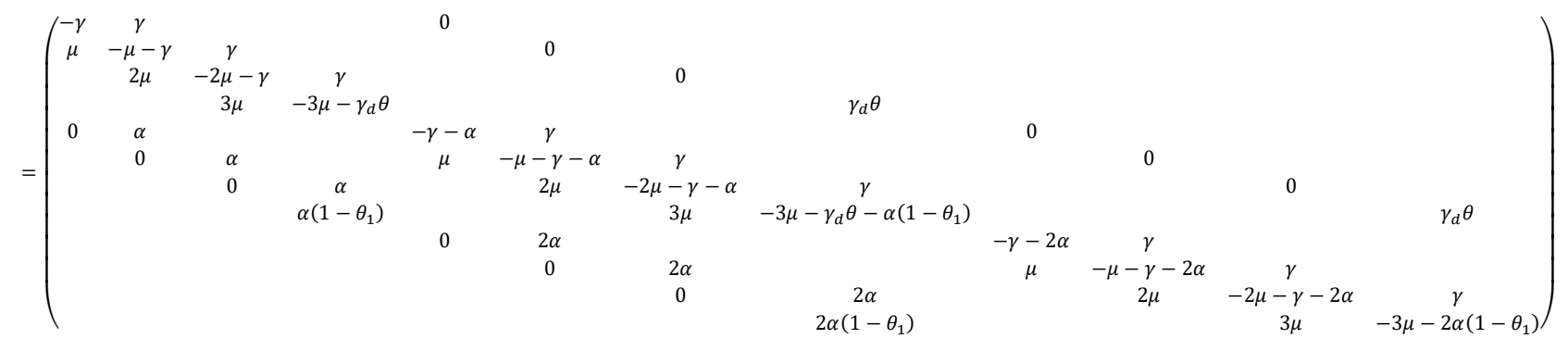

Balance equations can be expressed as follows 


$$
\begin{aligned}
& \left\{\begin{array}{c}
v_{0} Q_{1}^{(0)}+v_{1} Q_{2}^{(1)}=(0,0,0,0) \\
v_{0} Q_{0}^{(0)}+v_{1} Q_{1}^{(1)}+v_{2} Q_{2}^{(2)}=(0,0,0,0) \\
v_{1} Q_{0}^{(1)}+v_{2} Q_{1}^{(2)}=(0,0,0,0) .
\end{array}\right.
\end{aligned}
$$

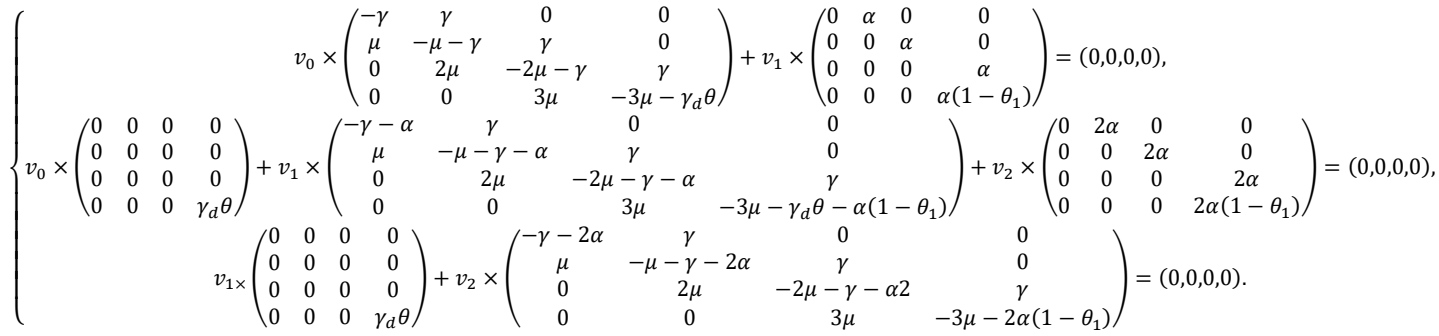

\section{Numerical Results}

Based on the blocking probability identified in the equation (8), (9), we proceed to graphically describe the change of the blocking probability depending on traffic load $(\rho)$, the number of wavelengths $(\omega)$, and the number of FDL. System model with parameters as follows: $\omega=16, L=2, \mu=0.020833, \theta=\theta_{1}=0.5$. Analysis results are also compared with simulation in some special cases. Similarly, the simulation parameters are used in [1], call $\beta=\rho / \omega$ is traffic load normalized per wavelength used at each output port (from 0.2 to $0.9(\operatorname{Erl}))$.

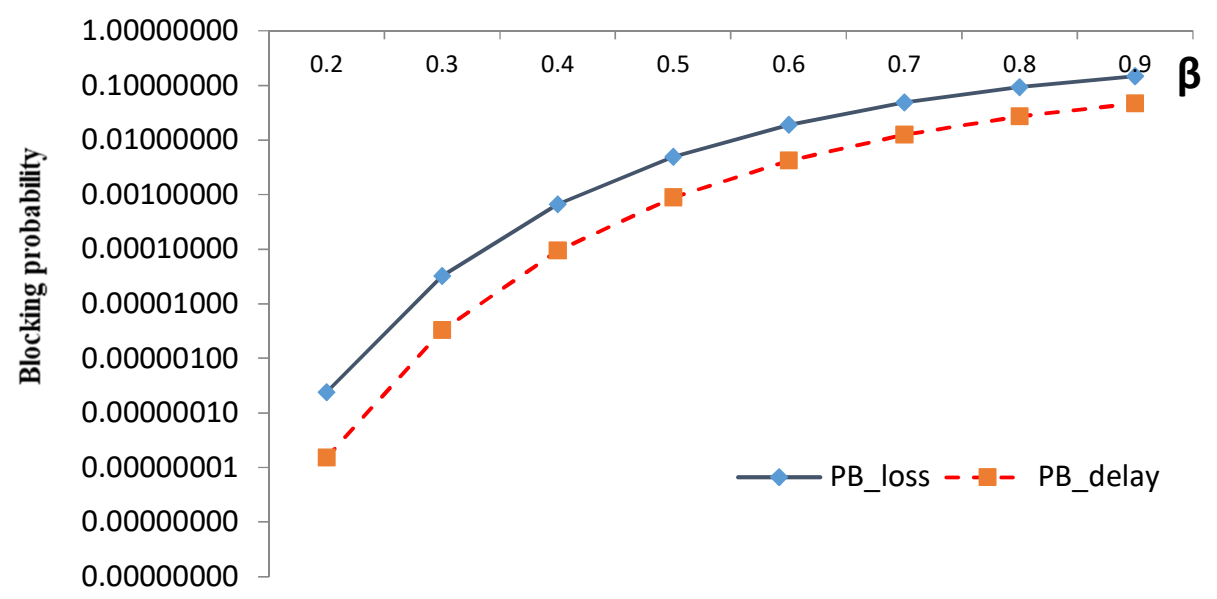

Figure 5. The blocking probabilities $P B_{\text {loss }}$ and $P B_{\text {delay }}$ vs $\beta$

Figure 5 shows the results of the blocking probability for the loss bursts and delay bursts $(\omega=16, L=2)$. In the analysis model, it is clear that the delay bursts have a smaller blocking probability due to delays in the FDL. 


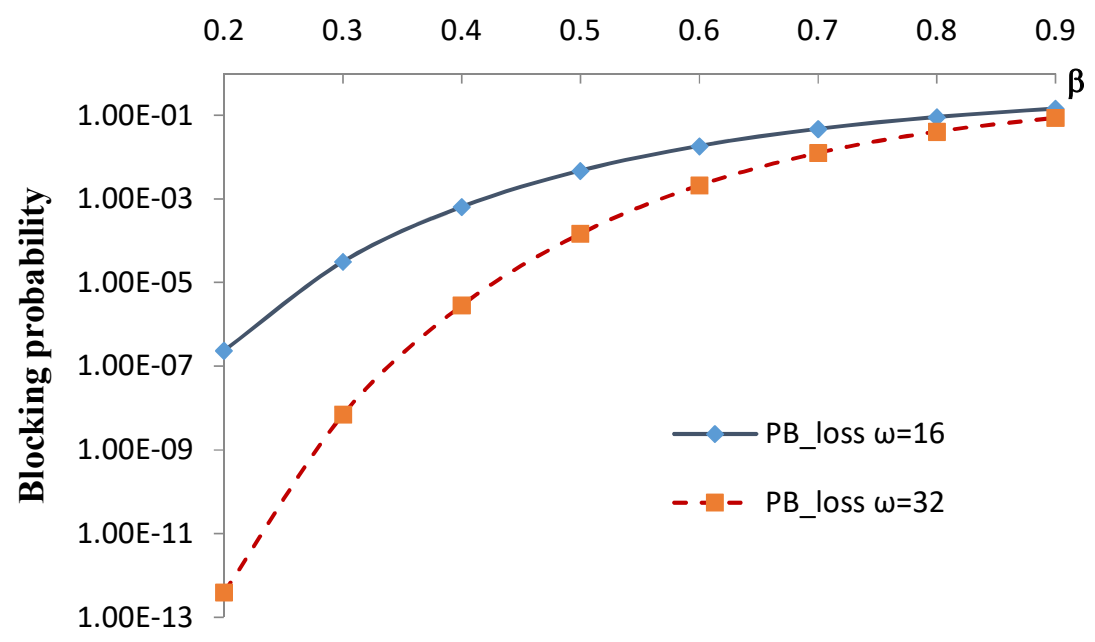

Figure 6a. The blocking probabilities $P B_{\text {loss }}$ vs $\beta$

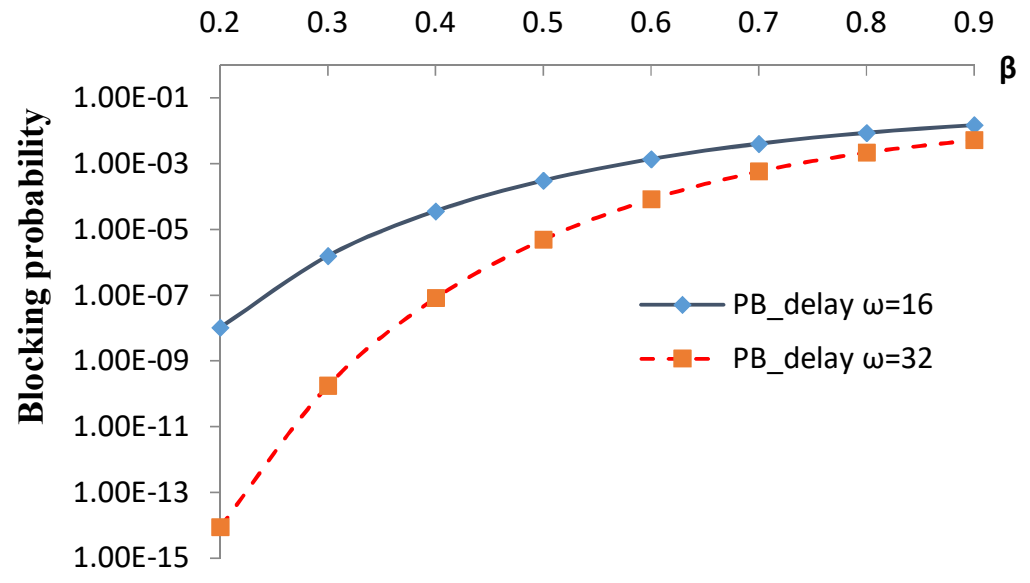

Figure $6 \mathrm{~b}$. The blocking probabilities $P B_{\text {delay }}$ vs $\beta$

Figure $6 \mathrm{a}$ and Figure $6 \mathrm{~b}$ show the blocking probability corresponding to the loss bursts and delay bursts of the Markov model with $\omega=16,32 ; L=2 ; \rho_{l}=0.3 \rho ; \rho_{d}=0.7 \rho$. Clearly, with a larger number of wavelengths, the blocking probability will be significantly improved, especially with low traffic loads. 


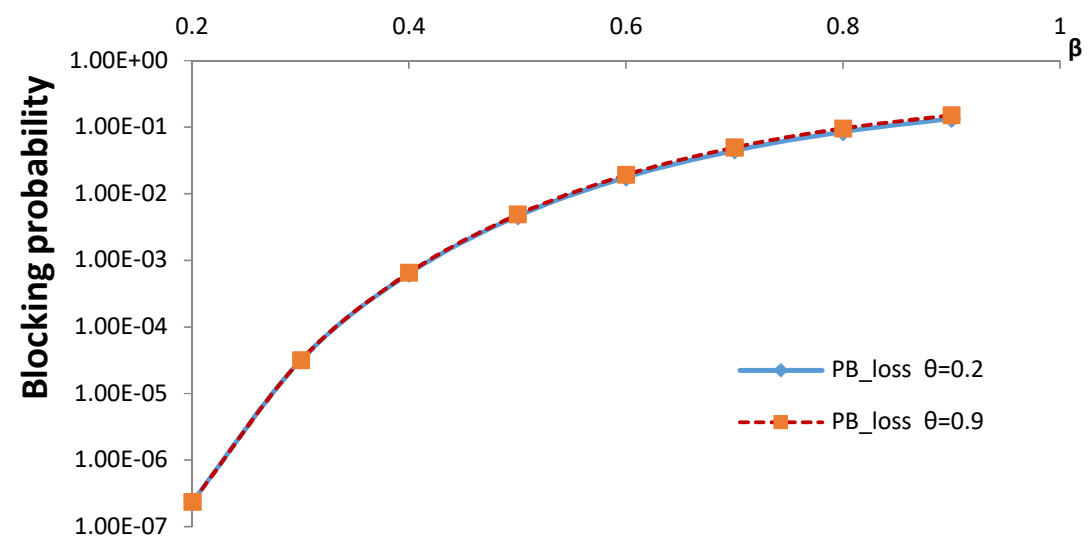

Figure 7a. The blocking probabilities $P B_{\text {loss }}$ with $\theta=0.2$ and $\theta=0.9$

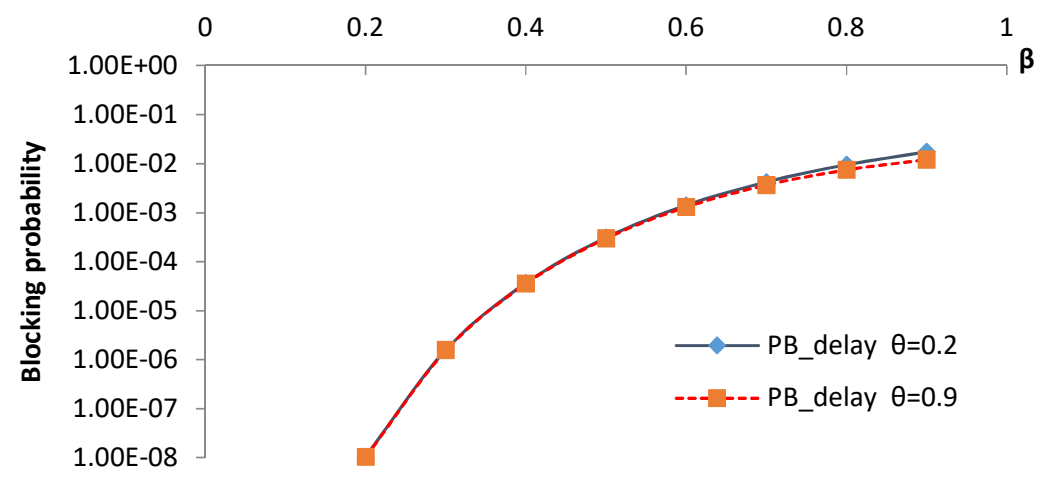

Figure $7 \mathrm{~b}$. The blocking probabilities $P B_{\text {delay }}$ with $\theta=0.2$ and $\theta=0.9$

Figure 7a shows that the blocking probability $P B_{\text {loss }}$ does not depend on the value $\theta$. This is perfectly consistent with the model we built above because of loss bursts in congestion condition of the system independent of the parameters $\theta$ and $\theta_{1}$. In contrast, with Figure $7 \mathrm{~b}$, when the value of $\beta$ is in the range of 0.2 to 0.9 , the probability retrial $\theta=$ 0.9 is always better than the probability retrial $\theta=0.2$.

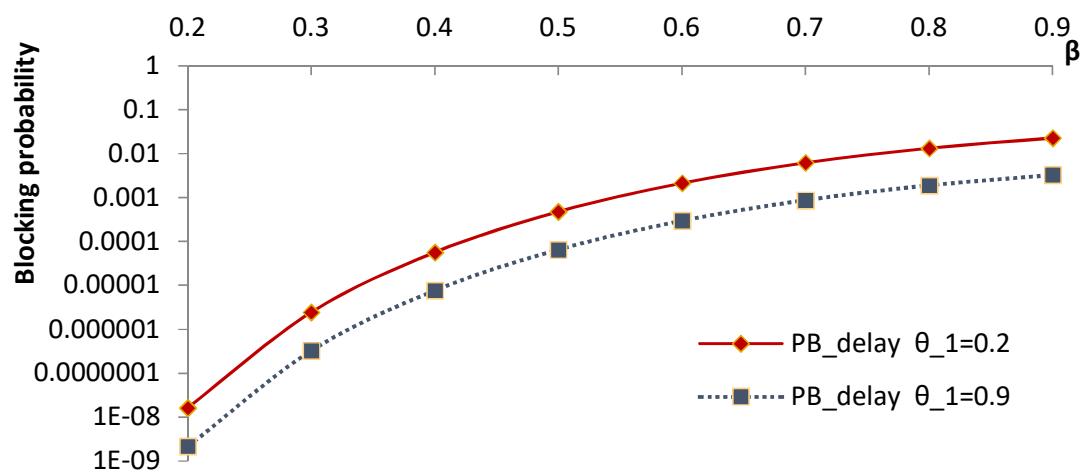

Figure 8a. The blocking probabilities $P B_{\text {delay }}$ with $\theta_{1}=0.2$ and $\theta_{1}=0.9$ 


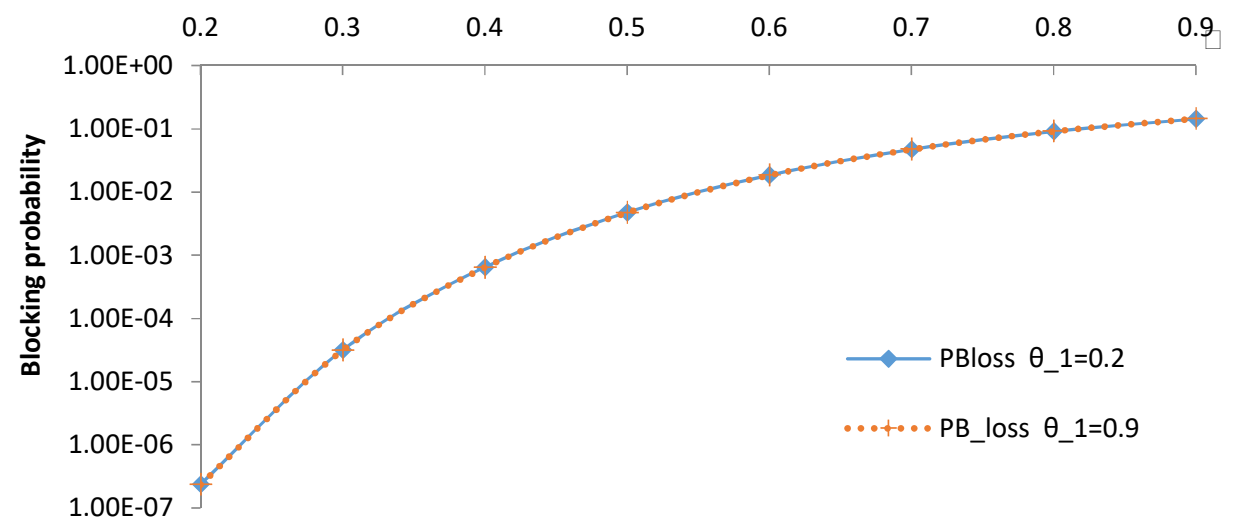

Figure $8 \mathrm{~b}$. The blocking probabilities $P B_{\text {loss }}$ with $\theta_{1}=0.2$ and $\theta_{1}=0.9$

The similar, Figure 8a when the value of $\beta$ is in the range of 0.2 to 0.9 , then the retrial probability $\theta_{1}=0.9$ is always better than the retrial probability $\theta_{1}=0.2$. In contrast, Figure $8 \mathrm{~b}$ shows that the congestion probability $P B_{\text {loss }}$ does not depend on the value $\theta_{1}$.

Figure 9 describes a comparison between the blocking probabilities of analytical model in this paper $\left(\theta=1.0, \theta_{1}=0.9\right)$ and the blocking probabilities of analytical model (i) in [1]. Clear that, our model in this paper has better results than in [1] due to using parameter $\theta_{1}$.

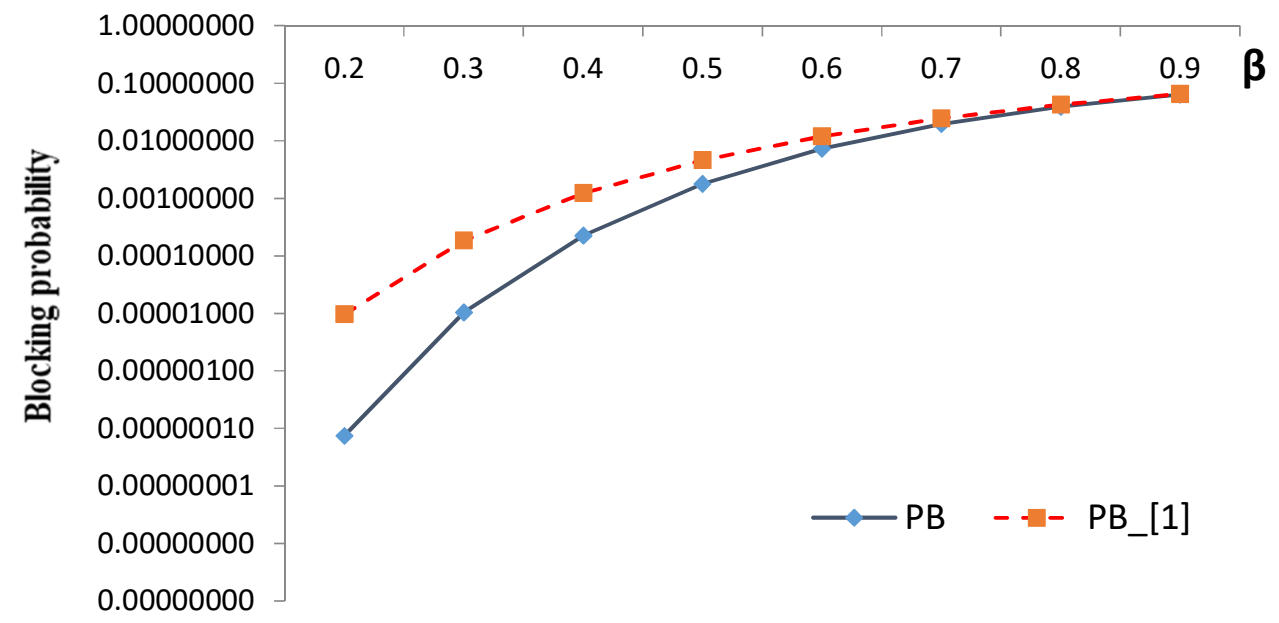

Figure 9. The blocking probabilities PB with $\theta=1.0, \theta_{1}=0.9$ and PB_[1]

We also implement a special case of simulation on NS-2 (use the OBS-ns Simulator) [12], in order to compare analytical results with simulation. Figure 10 shows that there is a good match between the analysis results and simulation. 


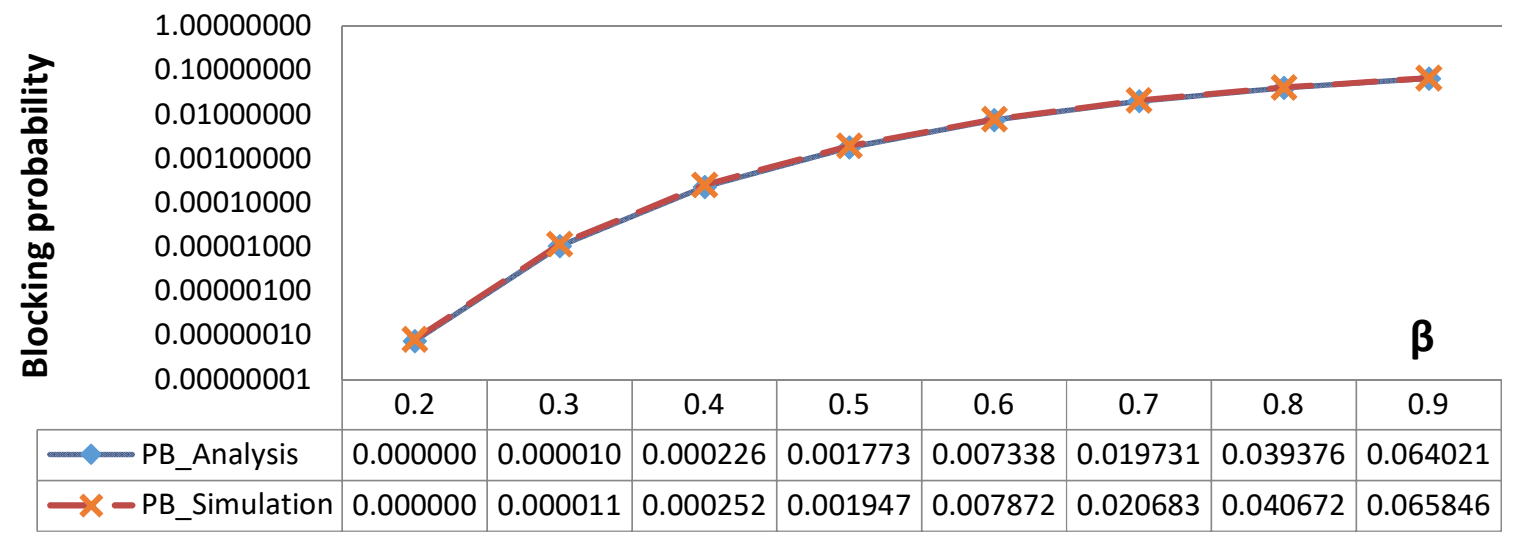

Figure 10. Blocking probabilities in the analytical case $\left(\theta=\theta_{1}=0.5\right)$ and simulation

\section{Conclusion}

The paper proposes a model to evaluate the performance of the SPL - feed-forward OBS architecture node with the retrial queuing model. Unlike previous studies [6], the proposed model considers the retrial factor for the FDL, i.e, considering that delayed traffic to FDL (orbit queue) with probability $\theta(\theta \leq 1)$ and retrial traffic (bursts come out from the FDL) when continuing the congestion at the output port can also re-circulate through one of the FDLs (2nd) with probability $\theta_{1}\left(\theta_{1}<1\right)$. Numerical results show that the proposed continuous-time Markov chain can be efficiently used to compute the blocking probabilities. The numerical results illustrate that the blocking probability strongly depends on the traffic load density, the number of used wavelengths and variant abilities of retrial probability.

Our future research plan will propose a model of traffic prediction based admission control with QoS [13], which includes a statistical method of forecasting the arriving rate of bursts for flexible wavelength allocation and a mechanism of prioritizing more resources for high-priority bursts.

\section{References}

[1] Chen Y, Qiao C, and Yu X (2004), Optical Burst switching: a new area in optical networking research, IEEE Network, vol. 18, no. 3, pp. 16-23. DOI: 10.1109/MNET.2004.1301018

[2] Vo Viet Minh Nhat, Le Van Hoa, Nguyen Hoang Son (2017), “A model of optimal burst assembly for delay reduction at ingress OBS nodes", Turkish Journal of Electrical Engineering \&amp; Computer Sciences, 3970-3982. DOI:10.3906/elk-1608-290

[3] Van Hoa Le, Viet Minh Nhat Vo, Manh Thanh Le (2018), "Throughput-based fair bandwidth allocation in OBS networks", ETRI Journal, Volume 40, Issue 5, Pages 624-633. https://doi.org/10.4218/etrij.2017-0253 
[4] Tien Van Do, Ram Chakka (2010), "A new performability model for queueing and FDL-related burst loss in optical switching nodes", Computer Communications 33 (2010) S146-S151. https://doi.org/10.1016/j.comcom.2010.04.030

[5] Akar Nail, and Sohraby Khosrow (2011), "Retrial Queueing Models of Multi-wavelength FDL Feedback Optical Buffers", IEEE Trans. Communications 59(10): 2832-2840. DOI: 10.1109/TCOMM.2011.071111.100521

[6] Dang Thanh Chuong (2016), "Analyzing the model of SPL \& feed-forward architecture at OBS core node with GI traffic", Research, Development and Application on Information\&Communication Technology, Vol V-2, No. 16 (36), pp. 58-66, ISDN 1859-3526. DOI: 10.15625/1813-9663/28/4/2353

[7] Conor McArdle, Daniele Tafani and Liam P. Barry (2011), "Analysis of a Buffered Optical Switch with General Interarrival Times", JOURNAL OF NETWORKS, VOL. 6, NO. 4, APRIL 2011. DOI:10.4304/jnw.6.4.536-548

[8] Akar Nail, Gunalayb Yavuz (2013), "Dimensioning shared-per-node recirculating fiber delay line buffers in an optical packet switch", Performance Evaluation (70) 1059-1071. https://doi.org/10.1016/j.peva.2013.09.003

[9] Daniele Tafani, (2012), "Analytic Modelling and Resource Dimensioning of Optical Burst Switched Networks", Doctor of Philosophy, School of Electronic Engineering, Faculty of Engineering and Computing, Dublin City University. DOI: 10.13140/RG.2.2.12436.09605

[10] Tien Van Do, Ram Chakka (2010), "An efficient method to compute the rate matrix for retrial queues with large number of servers", Applied Mathematics Letters 23, 638-643. https://doi.org/10.1016/j.aml.2010.02.003

[11] J. R. Artalejo and A. Gomez-Corral, "Retrial Queueing Systems". Springer, 2008. DOI: 10.1007/978-3-540-78725-9

[12] Network Simulator, At https://www.isi.edu/nsnam/ns/ (Last Access, October 2018)

[13] V. M. N. Vo, V. H. Le, H. S. Nguyen, and M. T. Le, "A model of QoS differentiation burst assembly with padding for improving the performance of OBS networks," Turkish J. Electr. Eng. Comput. Sci., vol. 26, no. 4, pp. 1783-1795, 2018. DOI: 10.26692/Surj/2017.12.56

\section{Copyright Disclaimer}

Copyright reserved by the author(s).

This article is an open-access article distributed under the terms and conditions of the Creative Commons Attribution license (http://creativecommons.org/licenses/by/3.0/). 